Pillai, N. C. \& Srinivasan, K. S. (1956). J. gen. Microbiol. 14, 248-255

\title{
The Amino Acid Metabolism of Aspergillus flavus
}

\author{
By N. C. PILlaI and K. S. SRINIVASAN \\ Department of Biochemistry, Indian Institute of Science, \\ Bangalore 3, India
}

SUMMARY: A complete quantitative amino acid analysis of the free amino acid fraction and the mycelial residue fraction of the mould Aspergillus flavus during different stages of growth has been made by the circular paper chromatographic technique. A qualitative analysis of the amino acid composition of the culture fluid has also been accomplished. The changes of the individual amino acids present in the various fractions on different days of incubation were studied and their implications discussed.

The introduction of elegant and specific methods of amino acid analysis (e.g. microbiological assay, paper chromatography) has accelerated the pace of research on the amino acid metabolism in moulds. Woolley \& Peterson (1936, $1937 a, b)$ isolated thirteen amino acids from Aspergillus sydowi and pointed out that only physical isolation was certain proof of the presence of any amino acid. Synthesis of aromatic amino acids, viz. tyrosine and tryptophan from inorganic $\mathrm{N}$ by $A$. niger, Zygorhyncus moelleri, Aspergillus oryzae, A. terreus and Penicillium flavo-glaucum was indicated by colorimetric tests (Skinner, 1934). Chromatography has been used to study the amino acid metabolism of $P$. chrysogenum (Narasimha Rao \& Venkataraman, 1952; Pyle, 1954).

In the present paper are presented the results of an investigation of the amino acid changes taking place in Aspergillus flavus during different stages of growth. The technique of circular paper chromatography (Giri \& Rao,1952 $a, b$ ) with different solvent systems (Rao \& Wadhwani, 1954, 1955) was used for the qualitative characterization and a quantitative estimation of most of the amino acids.

\section{METHODS}

Cultivation of the organism. A number of 11 . Pyrex conical flasks were plugged with non-absorbent cotton-wool and sterilized empty at $20 \mathrm{lb}$./sq.in. pressure for $30 \mathrm{~min}$. After cooling, $150 \mathrm{ml}$. of Czapek-Dox medium was distributed to each flask and sterilized at $10 \mathrm{lb}$./sq.in. pressure for $15 \mathrm{~min}$. A heavy spore suspension of Aspergillus flavus was inoculated into each of the flasks and incubated at room temperature $\left(26-28^{\circ}\right)$. The mycelia were separated from the culture liquids by filtration on a Buchner funnel. The culture fluids were stored in $250 \mathrm{ml}$. Erlenmeyer flasks in a refrigerator until analysis. The mycelia were washed free from culture fluid with minimal amounts of normal saline. The mycelial felts were squeezed between filter-papers to remove moisture and dried in a desiccator (over $\mathrm{P}_{2} \mathrm{O}_{5}$ ) to constant weight. 


\section{Preparation of samples}

Free amino acid fraction. Dry mycelium $(0.5$ g.) was ground with $10 \mathrm{ml}$. of $70 \%(\mathrm{v} / \mathrm{v})$ ethanol in water in a glass mortar for about $30 \mathrm{~min}$. The suspension was centrifuged and the clear supernatant collected in a conical flask. The solid residue was re-extracted thrice in the same manner. All the clear supernatants after centrifugation were pooled and evaporated under low pressure $(10 \mathrm{~mm}$. of mercury, $\left.50^{\circ}\right)$ to dryness and made up to $1 \mathrm{ml}$. with $10 \%(\mathrm{v} / \mathrm{v})$ isopropanol in water. The lots of mycelium harvested on different days were treated in the same manner, and the respective extracts were used for analysis of free amino acids.

Acid hydrolysates of mycelial residues. The residues left after extraction by $70 \%(\mathrm{v} / \mathrm{v})$ ethanol-water, were dried to constant weight in a desiccator and 0.2 g. samples hydrolysed by refluxing with $20 \mathrm{ml} .6 \mathrm{~N}-\mathrm{HCl}$ for $24 \mathrm{hr}$. The hydrolysate was evaporated repeatedly to dryness on a water-bath, with the addition of distilled water to remove the acid present in the solution. After evaporation to dryness the residue left was dissolved in $2 \mathrm{ml}$. of $10 \%(\mathrm{v} / \mathrm{v})$ isopropanol in water, allowed to stand for $15 \mathrm{~min}$. and then filtered. This filtrate was used for the amino acid analysis of the mycelial residue.

Culture fluids after growth. This was collected after the different periods of growth by filtration on a Buchner funnel. The fluids were stored under a layer of toluene in the refrigerator. For analysis $\mathbf{1 0 0 ~ m l . ~ s a m p l e s ~ o f ~ t h e s e ~ f l u i d s ~ w e r e ~}$ evaporated to dryness under low pressure, extracted with $95 \%(\mathrm{v} / \mathrm{v})$ acid ethanol (ethanol + water $+\mathrm{HCl}: 95+4 \cdot 5+0 \cdot 5$ ) to obtain salt-free amino acids (Baliga, Krishnamurthy, Rajagopalan \& Giri, 1955), evaporated to dryness and the residue made up to $2 \mathrm{ml}$. with $10 \%(\mathrm{v} / \mathrm{v})$ isopropanol in water. These solutions were used for the amino acid analysis of the culture fluids.

Nitrogen estimations of the three different fractions were made by the micro-Kjeldahl method.

Paper chromatography. The circular paper chromatographic technique developed by Giri \& Rao $(1952 a, b)$ was used for the identification of amino acids.

Two-dimensional chromatography by the ascending technique of Williams \& Kirby (1948) with butanol + acetic acid + water (40:10:50) and pyridine + water $(80: 20)$ as solvents for the first and second runs, respectively, was found useful for confirming the identity of some of the amino acids, e.g. $\gamma$-aminobutyric acid, tryptophan and other ninhydrin-positive substances not easily identified on the circular paper chromatogram.

Some of the amino acids were identified by specific tests carried out by the multiple sector technique on a circular chromatogram with the experimental solution spotted at the centre. Different sectors were cut from a completely irrigated and dried chromatogram and tested by either dipping in, or drawing through the appropriate reagent contained in a watch-glass. The special reagents were prepared according to Block, LeStrange \& Zweig (1952), Smith (1953) and Jepson \& Smith (1953).

The quantitative procedure for the estimation of amino acids was essentially the same as that described by Giri, Radhakrishnan \& Vaidyanathan (1952, 
1953). The modifications introduced by Rao \& Wadhwani (1955) regarding the solvent systems and temperature control for separation of amino acids were adopted with advantage.

\section{RESULTS}

Free amino acid composition of the mycelium. The amino acid composition of the ethanol-soluble fraction of the mycelium of Aspergillus flavus on different days of incubation is presented in Table 1. Most of the wide array of amino acids were at maximum concentration on the fifth day of incubation, with a subsequent decrease. The very high concentrations of alanine, glutamic and aspartic acids are interesting since aspartic and glutamic acids are known to be important parent substances for a number of other amino acids.

Table 1. Free amino acid composition of the mycelium of Aspergillus flavus on different days of incubation

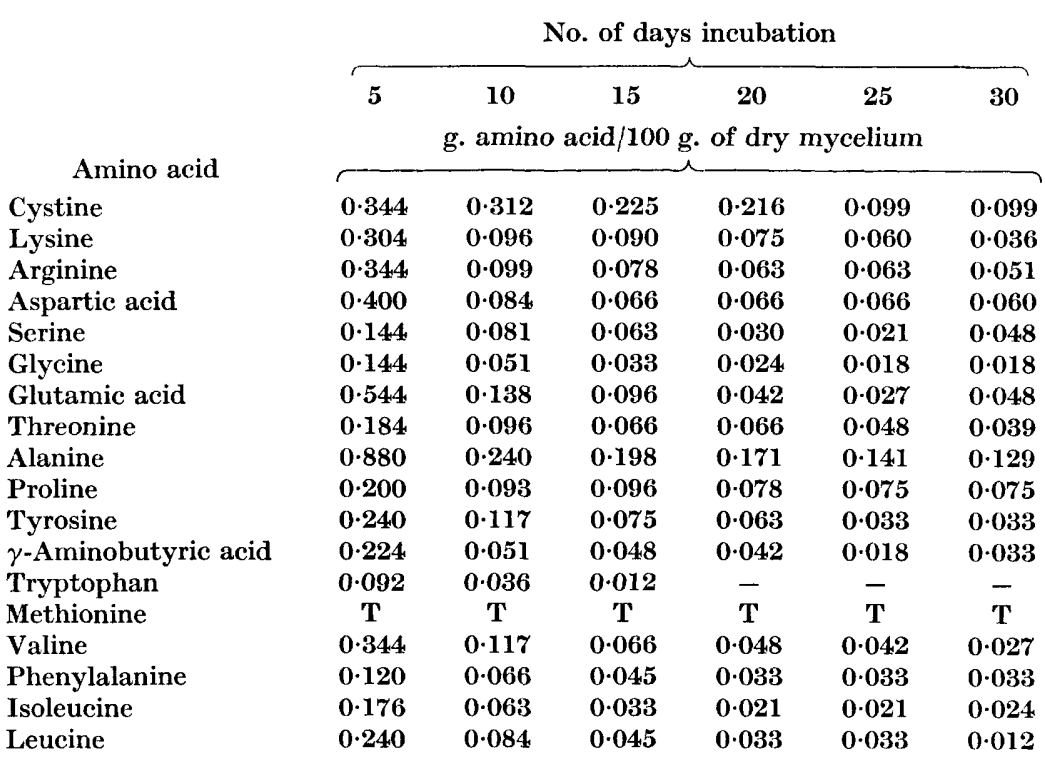

$$
\mathbf{T}=\text { present in traces only; }-=\text { absent. }
$$

Of the basic amino acids only lysine and arginine were present in high concentration; histidine was hardly detectable even by the highly sensitive Pauly's test. Lysine and arginine from a maximum concentration on the fifth day had dropped to a low value by the tenth day. The disappearance of these amino acids continued till the thirtieth day though at a slower rate.

Leucine, isoleucine, valine and glycine were at maximum concentration on the fifth day with a subsequent marked decrease by the tenth day. Valine showed the maximum change in concentration. The presence of proline in the free condition is worthy of note.

Of the aromatic amino acids, tryptophan and phenylalanine were in very low concentration, the amount of tyrosine being somewhat higher. Tryptophan had practically disappeared by the fifteenth day. 
Of the sulphur-containing amino acids cystine and methionine, methionine occurred only in traces in the free condition in Aspergillus flavus. Certain ninhydrin-positive spots occupying the positions of methionine sulphoxide, methionine sulphone and cysteic acid were, however, detected on two-dimensional chromatograms.

Table 2. Free amino acid composition of the mycelium of Aspergillus flavus on different days of incubation

Amino acid nitrogen as percentage of the total soluble nitrogen.

No. of days of incubation

\begin{tabular}{|c|c|c|c|c|c|c|}
\hline Amino acid & $\mathbf{5}$ & 10 & 15 & 20 & 25 & 30 \\
\hline Cystine & 4.99 & $13 \cdot 43$ & $12 \cdot 68$ & $15 \cdot 16$ & $9 \cdot 10$ & $9 \cdot 87$ \\
\hline Lysine & $7 \cdot 26$ & $6 \cdot 80$ & $8 \cdot 84$ & $8 \cdot 68$ & $9 \cdot 07$ & $5 \cdot 90$ \\
\hline Arginine & $13 \cdot 76$ & $11 \cdot 76$ & $12 \cdot 14$ & $12 \cdot 22$ & $15 \cdot 96$ & $14 \cdot 03$ \\
\hline Aspartic acid & $5 \cdot 25$ & $\mathbf{3} \cdot \mathbf{3 4}$ & $3 \cdot 36$ & $4 \cdot 19$ & $5 \cdot 48$ & $5 \cdot 41$ \\
\hline Serine & $2 \cdot 37$ & $3 \cdot 95$ & $4 \cdot 02$ & $2 \cdot 39$ & $2 \cdot 20$ & $5 \cdot 41$ \\
\hline Glycine & $\mathbf{3} \cdot \mathbf{3 4}$ & $3 \cdot 51$ & $2 \cdot 97$ & $\mathbf{2 \cdot 6 9}$ & $2 \cdot 65$ & $2 \cdot 87$ \\
\hline Glutamic acid & $6 \cdot 44$ & $4 \cdot 85$ & $4 \cdot 41$ & $2 \cdot 40$ & $2 \cdot 00$ & $3 \cdot 90$ \\
\hline Threonine & $2 \cdot 69$ & $4 \cdot 17$ & $3 \cdot 74$ & $4 \cdot 66$ & $4 \cdot 44$ & $3 \cdot 92$ \\
\hline Proline & $\mathbf{3} \cdot 81$ & $4 \cdot 17$ & $5 \cdot 65$ & $5 \cdot 73$ & $7 \cdot 18$ & $7 \cdot 79$ \\
\hline Tyrosine & $2 \cdot 31$ & $\mathbf{3} \cdot \mathbf{3 4}$ & $2 \cdot 80$ & $2 \cdot 93$ & $2 \cdot 00$ & $2 \cdot 18$ \\
\hline$\gamma$-Aminobutyric acid & $3 \cdot 79$ & $2 \cdot 56$ & $3 \cdot 16$ & $3 \cdot 43$ & $1 \cdot 91$ & $3 \cdot 82$ \\
\hline Tryptophan & 1.57 & $1 \cdot 83$ & $0 \cdot 80$ & - & - & - \\
\hline Methionine & $\mathbf{T}$ & $\mathrm{T}$ & $\mathbf{T}$ & $\mathbf{T}$ & $\mathbf{T}$ & $\mathbf{T}$ \\
\hline Valine & $5 \cdot 12$ & $4 \cdot 10$ & $3 \cdot 81$ & $3 \cdot 45$ & 3.95 & $2 \cdot 75$ \\
\hline Phenylalanine & $1 \cdot 27$ & $2 \cdot 07$ & $1 \cdot 84$ & $1 \cdot 68$ & $2 \cdot 25$ & $2 \cdot 38$ \\
\hline Isoleucine & $\mathbf{2 \cdot 3 4}$ & $2 \cdot 48$ & $1 \cdot 71$ & $1 \cdot 36$ & $1 \cdot 77$ & $2 \cdot 21$ \\
\hline Leucine & $3 \cdot 19$ & $\mathbf{3 \cdot 3 1}$ & $2 \cdot 32$ & $2 \cdot 13$ & $2 \cdot 79$ & $1 \cdot 10$ \\
\hline
\end{tabular}

$\mathbf{T}=$ present in traces only; $-=$ absent.

To assess the relative concentration of each amino acid in the mycelial extract, in Table 2 the nitrogen in each compound is expressed as a percentage of total soluble nitrogen. Irrespective of the duration of incubation, cystine, lysine and arginine were in a comparatively high concentration. The values for cystine, threonine and proline increased up to the twentieth day.

Amino acid composition of the mycelial residue. The amino acid composition of the ethanol-insoluble residue of the mycelium of Aspergillus flavus is given in Table 3, where the nitrogen of each amino acid is expressed as percentage of total nitrogen of the mycelial residue. The results show that the amino acid make-up of the mycelial protein varied during the period of incubation. From Table 3 it can be seen that the amount of bound glutamic acid in the protein was at a maximum in the fifteenth-day samples; alanine was at a maximum in the fifth-day sample. The concentration of these amino acids showed a decrease up to the twenty-fifth day after which there was a slight increase. Valine, tyrosine, leucine and isoleucine were minimum by about the twentieth day. There was a remarkably high concentration of lysine on the fifth day. After a subsequent steep fall in concentration till the tenth day there was again an increase till the twentieth day, and then a decline till the thirtieth day. It 
Table 3. Amino acid composition of ethanol-insoluble residue of the mycelium of Aspergillus flavus on different days of incubation

Amino acid nitrogen as percentage of total nitrogen of the mycelial residue.

\begin{tabular}{|c|c|c|c|c|c|c|}
\hline \multirow[b]{2}{*}{ Amino acid } & \multicolumn{6}{|c|}{ No. of days of incubation } \\
\hline & 5 & 10 & 15 & 20 & 25 & 30 \\
\hline Cystine & $4 \cdot 89$ & $9 \cdot 60$ & $10 \cdot 78$ & $9 \cdot 26$ & $9 \cdot 80$ & $9 \cdot 73$ \\
\hline Lysine & $28 \cdot 11$ & $10 \cdot 63$ & $12 \cdot 50$ & $22 \cdot 93$ & $17 \cdot 64$ & $12 \cdot 96$ \\
\hline Histidine & + & $\mathrm{T}$ & $\mathbf{T}$ & $\mathbf{T}$ & $\mathbf{T}$ & $\mathbf{T}$ \\
\hline Arginine & $9 \cdot 75$ & $6 \cdot 11$ & $6 \cdot 34$ & $5 \cdot 11$ & $6 \cdot 21$ & $6 \cdot 89$ \\
\hline Aspartic acid & $6 \cdot 69$ & $9 \cdot 16$ & $8 \cdot 77$ & 7.07 & $11 \cdot 70$ & $13 \cdot 64$ \\
\hline Serine & $3 \cdot 62$ & $5 \cdot 86$ & $7 \cdot 41$ & $6 \cdot 13$ & $6 \cdot 00$ & $5 \cdot 21$ \\
\hline Glycine & $4 \cdot 74$ & $6 \cdot 79$ & $6 \cdot 50$ & $7 \cdot 06$ & $6 \cdot 57$ & $6 \cdot 31$ \\
\hline Glutamic acid & $4 \cdot 28$ & $5 \cdot 86$ & $7 \cdot 36$ & $5 \cdot 70$ & $6 \cdot 27$ & $6 \cdot 12$ \\
\hline Threonine & $3 \cdot 10$ & $5 \cdot 03$ & $4 \cdot 81$ & $3 \cdot 88$ & $3 \cdot 61$ & $4 \cdot 11$ \\
\hline Alanine & $7 \cdot 84$ & $6 \cdot 47$ & $6 \cdot 43$ & $5 \cdot 95$ & $5 \cdot 00$ & $5 \cdot 85$ \\
\hline Proline & $3 \cdot 81$ & $5 \cdot 97$ & $4 \cdot 97$ & $4 \cdot 76$ & $4 \cdot 23$ & $3 \cdot 98$ \\
\hline Tyrosine & $3 \cdot 40$ & $4 \cdot 90$ & $2 \cdot 70$ & $1 \cdot 89$ & $2 \cdot 99$ & $3 \cdot 66$ \\
\hline Methionine & $\mathbf{T}$ & $\mathbf{T}$ & $\mathbf{T}$ & $\mathrm{T}$ & $\mathbf{T}$ & + \\
\hline Valine & $3 \cdot 51$ & $6 \cdot 06$ & $5 \cdot 98$ & $4 \cdot 82$ & $5 \cdot 03$ & $7 \cdot 02$ \\
\hline Phenylalanine & $1 \cdot 49$ & $1 \cdot 88$ & $1 \cdot 80$ & $1 \cdot 14$ & $1 \cdot 06$ & $1 \cdot 05$ \\
\hline Isoleucine & $3 \cdot 45$ & $3 \cdot 04$ & $2 \cdot 75$ & $2 \cdot 22$ & $2 \cdot 31$ & $2 \cdot 29$ \\
\hline Leucine & $4 \cdot 38$ & $3 \cdot 72$ & $3 \cdot 73$ & $3 \cdot 39$ & $3 \cdot 52$ & $3 \cdot 49$ \\
\hline
\end{tabular}

$\mathbf{T}=$ present in traces only; $+=$ present.

was further observed that the amount of arginine decreased from a maximum concentration on the fifth day till the twentieth day and then showed signs of increase till the thirtieth day. The concentration of aspartic acid, however, was found to be minimum by the fifth day. After attaining a secondary minimum on the twentieth day the concentration rose sharply reaching a maximum by the thirtieth day.

Amino acid composition of culture fluids. The amino acid composition of culture fluids is presented in Table 4. As the amino acid concentration was

Table 4. Amino acids in the culture medium of Aspergillus flavus on different days of incubation

\begin{tabular}{lcccccc}
\multicolumn{7}{c}{ No. of days of incubation } \\
\cline { 2 - 5 }$\quad$ Amino acid & $\mathbf{5}$ & $\mathbf{1 0}$ & $\mathbf{1 5}$ & $\mathbf{2 0}$ & 25 & 30 \\
Lysine & - & - & - & - & - & + \\
Aspartic acid & + & + & + & + & + & ++ \\
Glycine & ++ & ++ & ++ & ++ & ++ & + \\
Serine & + & + & - & $\mathbf{T}$ & + & + \\
Glutamic acid & $\mathbf{T}$ & $\mathbf{T}$ & $\mathbf{T}$ & $\mathbf{T}$ & + & ++ \\
Alanine & + & - & - & - & - & + \\
Tyrosine & - & - & $\mathbf{T}$ & $\mathbf{T}$ & $\mathbf{T}$ & + \\
Leucines & - & + & $\mathbf{T}$ & - & - & + \\
A & - & - & - & + & + & ++ \\
B & - & + & - & - & - & - \\
C & - & + & - & - & - & -
\end{tabular}

$++=$ very prominent $;+=$ present $\mathrm{T}=$ present in traces only; $-=$ absent; $\mathrm{A}, \mathrm{B}$ and $\mathrm{C}$ are three ninhydrin-positive unidentified substances. 
too low to be measured, and as it assumed a yellowish brown colour as the organism was being incubated, this study was restricted to a qualitative examination. Of the eight amino acids detected (Table 4) aspartic acid and glycine occurred throughout the period and were at a maximum on the thirtieth day. Aspartic acid and glycine were in a much higher concentration as compared to the other amino acids in the culture fluids at all times. There was as well a direct correlation between the disappearance of tyrosine, tryptophan, phenylalanine and the development of colour in the medium. Three unidentified ninhydrin-positive compounds in the culture fluids were also detected on the two-dimensional chromatograms.

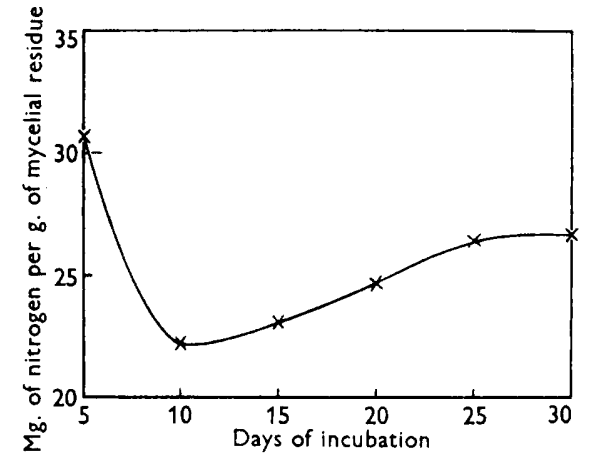

Fig. 1

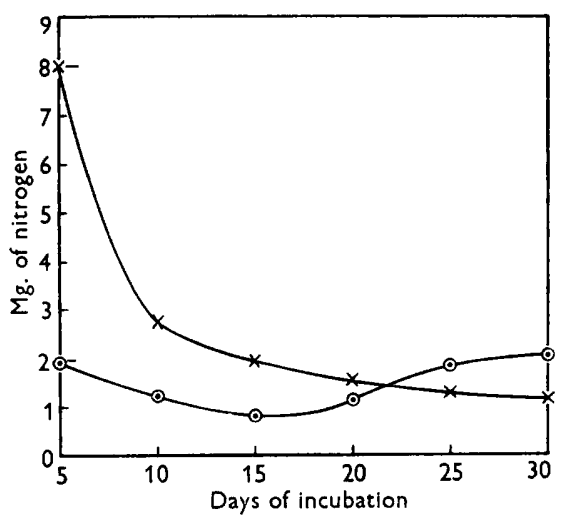

Fig. 2

Fig. 1. Changes in the nitrogen content of the ethanol-insoluble residue of the mycelium of Aspergillus flavus, on different periods of incubation. $\times-\times, \mathrm{mg}$. of nitrogen/g. of mycelial residue.

Fig. 2. Changes in the nitrogen content of the ethanol-soluble fraction of the mycelium of Aspergillus flavus $(\times-\times)$, and the changes in the total nitrogen content of the culture fluid of $\boldsymbol{A}$. flavus $(\odot-\odot)$ ) on different periods of incubation. $\times-\times$, mg. of soluble nitrogen $/ \mathrm{g}$. of dry mycelium; $\odot-\odot, \mathrm{mg}$. of total nitrogen $/ 100 \mathrm{ml}$. of the culture fluid.

The changes in the total nitrogen content of the ethanol-insoluble residue, ethanol-soluble fraction and the culture fluid of Aspergillus flavus on different periods of incubation are given in Figs. 1 and 2. The nitrogen content of the culture fluid was found to be much lower than in the other two fractions. The changes in concentration of nitrogen in the two fractions of the mycelium were more marked than in the culture fluid.

The total dry weight of the mycelium on different periods of incubation did not show any appreciable variation. However, it showed a slight increase from the fifth day to the fifteenth day, whereafter there was a decrease up to the thirtieth day of incubation.

\section{DISCUSSION}

The occurrence of high concentrations of amino acids in the ethanolic extracts of the fifth-day sample of the mycelium indicates that in the young mycelium there is intense metabolic activity, the amino acids synthesized being either 
directly incorporated into the protein of the cells or used as a source of nitrogen for the synthesis of all the nitrogenous cellular constituents. From a consideration of the nitrogen content of both the ethanol-soluble and ethanol-insoluble fractions (Figs. 1, 2), it can be stated that the mycelium contains nearly $50 \%$ more protein before prolific sporulation (fifth day) than after prolific sporulation.

The hydroxy amino acids serine and threonine were present in high concentration in bound form in the mycelial protein (Table 3), whereas their content in the ethanolic extract was low. This might be due to the efficient incorporation of these amino acids as soon as formed into the cellular protein. Information regarding the decarboxylation of amino acids by fungi is scanty. The detection of $\gamma$-aminobutyric acid, therefore, in the ethanolic extracts is interesting.

In contrast to the amino acid content of the mycelium, the culture fluid contained the maximum concentration of amino acids on the thirtieth day (Figs. 1, 2). This general increase in the concentration of amino acids and also of total nitrogen in the culture fluid (Fig. 2) towards the thirtieth day may be attributed to the proteolytic activity of the mycelium.

We wish to thank Professor K. V. Giri, Head of the Department of Biochemistry, for his kind guidance and keen interest in the work.

\section{REFERENCES}

Baliga, B. R., Krishnamurthy, K., Rajagopalan, R. \& Giri, K. V. (1955). A simple method for desalting biological fluids for chromatography. J. Indian Inst. Sci. 37, no. 1, 18.

Block, R. J., LeStrange, R. \& Zweig, G. (1952). Paper Chromatography, A Laboratory Manual. New York: Academic Press.

Giri, K. V. \& Rao, N. A. N. (1952a). A technique for the identification of amino acids separated by circular paper chromatography. Nature, Lond., 169, 923.

Giri, K. V. \& RAo, N. A. N. (1952b). Circular paper chromatography. I. A technique for the separation and identification of amino acids. J. Indian Inst. Sci. 34, 95.

Giri, K. V., Radhakrishnan, A. N. \& Vaidyanathan, C. S. (1952). Some factors influencing the quantitative determination of amino acids separated by circular paper chromatography. Analyt. Chem. 24, 1677.

Giri, K. V., Radhakrishnan, A. N. \& Vaidyanathan, C. S. (1953). Circular paper chromatography. Part VI. The quantitative determination of amino acids. J. Indian Inst. Sci. 35, 145.

Jepson, J. B. \& Smith, I. (1953). Multiple dipping procedures in paper chromatography: specific test for hydroxyproline. Nature, Lond., 172, 1100.

Narasimha Rao, P. L. \& Venkataraman, R. (1952). Nitrogen metabolism of Penicillium chrysogenum-Q176. Experientia, 8/9, 350.

Pyle, A. J. (1954). Some aspects of the amino acid metabolism of Penicillium chrysogenum. J. gen. Microbiol. 11, 191.

RaO, N. A. N. \& WADHWANi, T. K. (1954). Resolution of mixtures of amino acids, by circular paper chromatography. Curr. Sci. 23, 359.

Rao, N. A. N. \& Wadhwani, T. K. (1955). Quantitative estimation of amino acids by circular paper chromatography. J. Indian Inst. Sci. 37, 130.

SkInNER, C. E. (1934). The synthesis of aromatic amino acids from inorganic nitrogen by moulds and the value of mould proteins in diets. J. Bact. $28,95$. 
SмIтн, I. (1953). Colour reactions on paper chromatograms by a 'dipping technique'. Nature, Lond., 171, 43.

Williams, R. J. \& Kirby, H. (1948). Paper chromatography using capillary ascent. Science, 107, 481.

Woolley, D. W. \& Peterson, W. H. (1936). The chemistry of mould tissue. XI. Isolation of leucine and isoleucine from Aspergillus sydozi. J. biol. Chem. 114, 85.

Wooliey, D. W. \& Peterson, W. H. (1937 a). The chemistry of mould tissue. XII. Isolation of arginine, histidine and lysine from Aspergillus sydowi. J. biol. Chem. 118, 363.

Woolley, D. W. \& Peterson, W. H. (1937b). The chemistry of mould tissue. XIII. Isolation of monoaminomonocarboxy- and some monoaminodicarboxyacids from Aspergillus sydowi. J. biol. Chem. 121, 507.

(Received 24 June 1955) 